\title{
Psychological impact of the COVID-19 pandemic on medical and rehabilitation sciences university students in Saudi Arabia
}

\author{
Hassan Izzeddin Sarsak* \\ Occupational Therapy Program, Batterjee Medical College, Jeddah, Saudi Arabia \\ E-mail: hassan.sarsak@bmc.edu.sa.
}

Received 10 February 2021

Accepted 19 October 2021

\begin{abstract}
.
BACKGROUND: The COVID-19 pandemic has been associated with increased stressful impact among different populations that are at an increased risk of mental health problems, such as university students.

OBJECTIVES: To investigate the psychological effects of the COVID-19 pandemic on medical and rehabilitation sciences university students, explore the factors affecting them, and provide helpful recommendations for better mental health.

METHODS: An online survey was conducted with a sample of medical and rehabilitation sciences students. The anxiety visual analogue scale, the perceived stress scale (PSS), and the impact of events scale-revised (IES-R) were applied.

RESULTS: A total of 244 students completed the survey (60.7\% were female). The mean scores of total anxiety, PSS and IES-R were significantly higher in females than males. $34.5 \%$ and $29.4 \%$ of the students reported that their sleep and appetite were respectively disturbed during the pandemic. Students with adequate knowledge about the COVID-19 pandemic reported a significant decrease in anxiety level $(p=0.01)$, perceived stress $(p=0.00)$, and less negative impacts of the pandemic $(p=0.01)$.

CONCLUSION: This study shed light on factors that play a major role in anxiety, perceived stress and stressful impact of COVID-19 pandemic among medical and health sciences students, such as gender, knowledge, educational level, presence of chronic condition, and family financial status. Developing proper mental health management guidelines and providing psychological support are recommended and should be considered.
\end{abstract}

Keywords: Anxiety and perceived stress, coronavirus, medical and rehabilitation sciences students, mental health, psychological support

\section{Introduction}

As the coronavirus pandemic rapidly sweeps across the world, it is inducing a significant negative impact and considerable degree of mass hysteria, economic burden and financial losses, fear, worry and concern in public, and burnout among certain vulnerable groups in particular, such as older adults, healthcare students and healthcare workers (HCWs), and individuals with chronic health conditions [1-4]. The main reported adverse psychological impacts and mental symptoms to date at both pre-infection and post-infection periods are elevated rates of emotional distress and various psychological disturbances, such as stress or anxiety (i.e., developing signs and symptoms of obsessive compulsive and/or post-traumatic stress disorders), panic attacks, frustration, uncertainty, financial stress, distorted usual activities of daily living and routines, higher levels of loneliness and depression, insomnia, harmful drug use, selfharm or suicidal behaviors, and destructive unhealthy maladaptive behaviors, such as drinking alcohol and smoking [5-8]. Issues of service access and continuity for individuals with mental health conditions 
have been on the rise and are also now a major concern along with the mental health and well-being of frontline workers [9, 10]. The COVID-19 pandemic has been associated with increased stressful impact among general population as reported at different countries worldwide [11]. A recent study found that throughout the early stage of the COVID-19 outbreak in Saudi Arabia, the results showed that nearly one-fourth of the general population experienced moderate to severe psychological impact [12].

The time of the COVID-19 pandemic can be emotionally challenging and stressful to all persons affected, and in particular those groups of the population that are at an increased risk of mental health problems, such as university students [13]. COVID-19 pandemic has been disrupting the students' educational process. Millions of students and educators have been affected by school closures and other restrictions. Most governments around the world closed educational institutions in an attempt to contain the spread of the COVID-19 pandemic. These closures impacting over $60 \%$ of the world's student population. In response, many governments provided schools and university students with teaching and learning alternatives utilizing online and distance learning. Although precautions were taken, the students' learning process was negatively affected especially when it comes to teaching and learning practical handson skills. Students who have been stripped of their freedom due to COVID-19 are deeply concerned about when face to face education will happen [14]. A recent study reported that during the COVID19 pandemic, more than one fifth of junior high and high school students' mental health was negatively affected [15]. More recent studies conducted on medical and rehabilitation sciences students during the pandemic report that they show high levels of emotional distress as they have a greater risk of interacting with COVID-19 patients and they might lack adequate knowledge [16]. Poor knowledge can lead to misconceptions and high levels of stress and anxiety [17]. In Saudi Arabia, the COVID-19 pandemic situation has affected college and university students mental health with increased stress, anxiety and depressive thoughts due to the COVID-19 outbreak and stay-at-home orders. Before the pandemic, students used to attend most classes in-person and socialize on-campus regularly. During the pandemic, major changes in their regular daily routines disrupted their lifestyle. Multiple stressors with negative impacts have been affecting some of the students mental and emotional health, such as fear and worry about their own health and of their loved ones, difficulty in concentrating, disruptions to sleeping patterns, decreased social interactions due to isolation and physical distancing, increased concerns on academic performance with shifting to remote education and adoption of negative behaviors and coping mechanisms [8, 12].

The purpose of this study was to investigate the psychological effects of the COVID-19 pandemic on medical and rehabilitation sciences university students, the stress they perceived, the factors affecting them, and their knowledge about COVID-19. In addition, we aim to enhance medical and rehabilitation sciences university students awareness and knowledge related to COVID-19 pandemic through providing helpful and useful recommendations for developing proper mental health management guidelines and providing psychological support and psychosocial crisis prevention and counseling services. We hypothesize that (1) medical and rehabilitation sciences students are highly anxious about being infected with COVID-19, therefore, they are at increased risk of developing psychological and mental health issues during the COVID-19 crisis, and (2) junior students enrolled in the preclinical stages have less knowledge than their seniors enrolled in the clinical stages, hence will have a significant higher levels of anxiety and stress.

\section{Methods}

\subsection{Study design and participants}

This is a descriptive cross-sectional research study design, applying survey methodology. At the beginning of December (2020), when the data was collected, distance education at universities was underway and strict instructions related to COVID-19 pandemic were imposed. Any medical and rehabilitation sciences student who had been enrolled in the medical school was eligible to participate. Anyone below 18 years of age, or unwilling or unable to give informed consent was excluded. Prospective data were collected over four consecutive weeks commencing in December 2020 as students were finishing up their semester workload and preparing for the final exams. Data capture was undertaken using a self-reported, online survey tool. This study was approved from the medical college research and ethical committee. Consent forms were obtained from all participants. 


\subsection{Survey instruments}

In the beginning of the survey, a brief description was provided and participant's consent was obtained. The first part of the survey included questions about the participants demographics. The second part included 15 questions exploring psychological impact of the COVID-19 pandemic on medical and rehabilitation sciences university students, their stress levels, COVID-19 related knowledge (i.e., ways of transmission, signs and symptoms, preventive methods, infection control, protective measures), and whether or not they need support from the university in regards to their university workload and areas of those needs. To asses anxiety of being infected with COVID-19, we used a single 10 pointed ordinal visual analogue scale ranging from 0 (not at all anxious) to 10 (extremely anxious).

\subsubsection{Perceived Stress Scale (PSS)}

COVID-19 related stress was assessed using PSS. It consists of 10 items scored between 0 (never) and 4 (very often). Individual scores on the PSS can range from 0 to 40 with higher scores indicating higher perceived stress [18]. The PSS is an easy-to-use questionnaire with established acceptable psychometric properties. It is a precise measure of personal stress and helps measure individual stress levels and understand how different situations affect our feelings and our perceived stress [19].

\subsubsection{Impact of Events Scale-Revised (IES-R)}

The psychological impact of COVID-19 was examined using the IES-R. It measures how much the person has been bothered after a traumatic stressful event using a 5-point scale ranging from 0 (not at all) to 4 (extremely). The IES-R consists of 22 items with a score range of $0-88$. Scores higher than 24 are of concern and the higher the score the greater the concern for post-traumatic stress disorder (PTSD) and associated health and well-being consequences. It has proven to be a helpful and useful tool in determining how distressing difficulties have been during a stressful event [20].

\subsection{Statistical analysis}

Descriptive statistics were calculated for all variables with frequency and percentage for categorical variables and means and standard deviation (SD) for continuous variables. Association of presence of anxiety and categorical variables was analyzed with the
Chi-square $\left(\chi^{2}\right)$ test and one-way analysis of variance (ANOVA) was used for continuous variables. The statistical Package for Social Sciences (SPSS) version 22.0 was used and all significant tests were two-tailed with $p<0.05$ accepted for statistical significance.

\section{Results}

\subsection{Participants demographics}

A total of 244 students participated and filled the survey. Students were from different programs and educational levels. About $60.7 \%$ of the participants $(n=148)$ were female, $39.3 \%(n=96)$ were male and the mean age was $22.10 \pm 2.69$ (see Table 1).

\subsection{Participants responses}

Students responses to the questions of whether they were anxious about becoming infected with COVID-19, the PSS, the IES-R, and the factors affecting them are respectively represented in Table 2. Chronic condition was found to be a significant factor that increased anxiety $(p=0.01)$. The current student's family income below the minimum monthly wage compared to prior the pandemic increased anxiety significantly $(p=0.02)$. COVID-19 proper related knowledge lowered anxiety significantly $(p=0.01)$. Anxiety in female students was significantly higher than in male students $(p=0.03)$. Preclinical students (the first 2-3 years) reported a significant increase in anxiety compared to students at the clinical stage of their studies (the last 3-5 years) $(p=0.01)$. A significantly higher level of perceived stress was found with students whose family's income was below the

Table 1

Participants' demographics $(N=244)$

\begin{tabular}{lcc}
\hline Demographic & $n$ & $\%$ \\
\hline Gender & & \\
$\quad$ Female & 148 & $60.7 \%$ \\
$\quad$ Male & 96 & $39.3 \%$ \\
Age (mean, SD) & $22.10 \pm 2.69$ & \\
Program & & \\
Occupational Therapy (OT) & 13 & $5.3 \%$ \\
Medicine & 42 & $17.2 \%$ \\
Physical Therapy (PT) & 45 & $18.5 \%$ \\
Dentistry & 37 & $15.2 \%$ \\
Healthcare Administration (HA) & 14 & $5.7 \%$ \\
Radiology & 15 & $6.1 \%$ \\
Pharmacy & 18 & $7.4 \%$ \\
Nursing & 28 & $11.5 \%$ \\
Respiratory Therapy (RT) & 32 & $13.1 \%$ \\
\hline
\end{tabular}


Table 2

Anxiety, perceived stress, and impact of events scales total scores in relation to different factors $(N=244)$

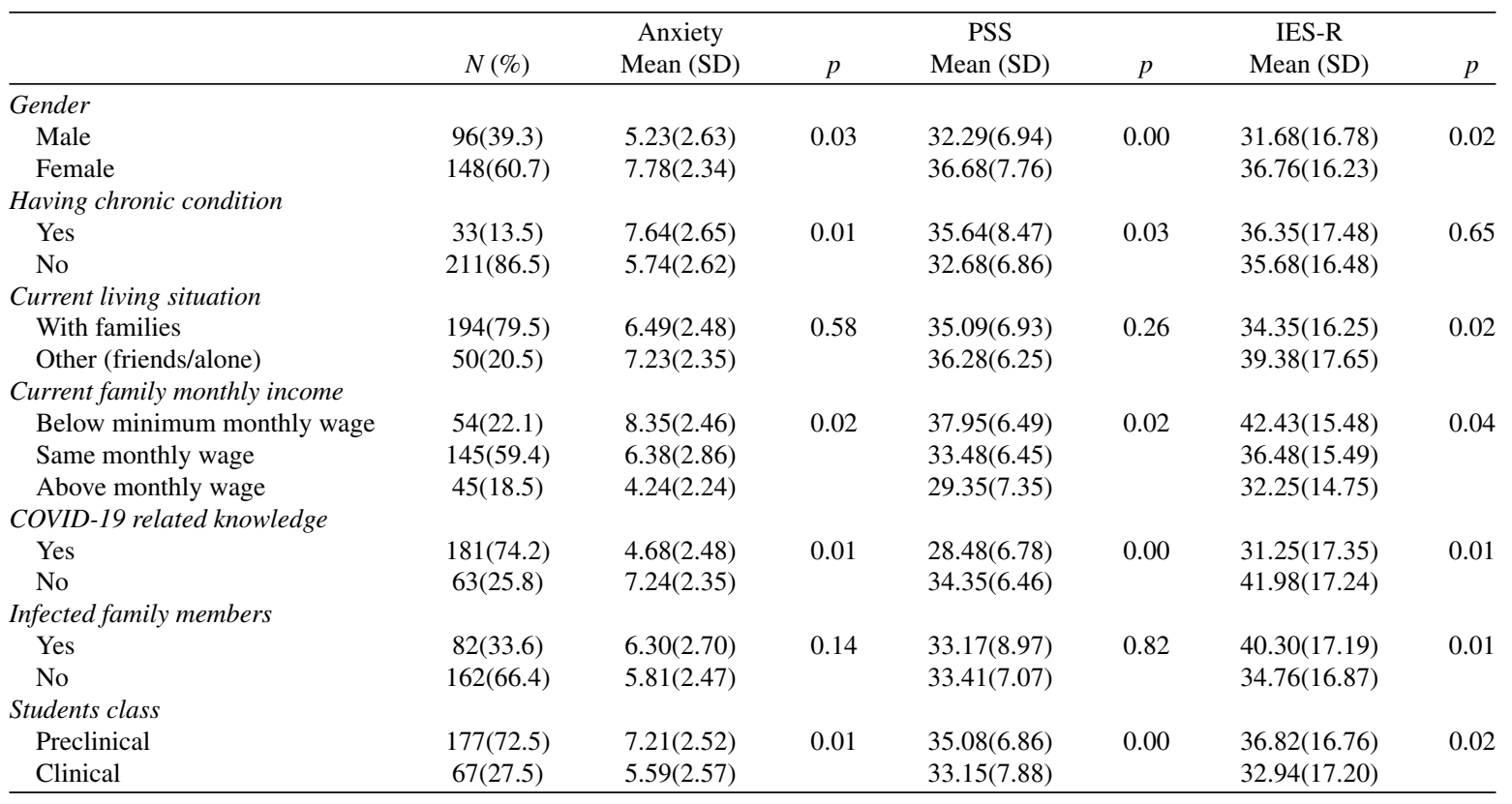

minimum monthly wage compared to prior the pandemic $(p=0.02)$. The perceived stress level in female was higher than in male $(p=0.00)$. Having a chronic condition was found to be a significant factor that increased perceived stress among students $(p=0.03)$. Perceived stress was significantly higher among students who stated that they have poor knowledge about COVID-19 $(p=0.00)$. The PSS scores were significantly higher among junior preclinical students compared to senior clinical students $(p=0.00)$. The average scores given to IES-R in female were significantly higher $(p=0.02)$. At the time of the survey, IES-R scores of those living with their family were significantly lower than the others living with friends or living alone $(p=0.02)$. Significant higher IES-R scores were found among students who had relatively poor COVID-19 related knowledge $(p=0.01)$. Having infected family members with COVID-19 led to a significant increase in the IES-R scores $(p=0.01)$. There was a significant difference between the students' levels in the IES-R scores with preclinical being significantly higher $(p=0.02)$.

At the time of COVID-19, the students sleep patterns changed as $34.5 \%(n=84)$ reported sleeping late and waking up more frequently. $29.4 \%(n=72)$ of the students stated that their appetite during COVID-19 became worse with irregular times of food intake. Students reported getting COVID-19 related information from different resources with $45.6 \%$ relying on data obtained from the official documents released from the ministry of health, $22 \%$ kept track of official statements of the world health organization (WHO), $33 \%$ followed national television channels, and more than $66.2 \%$ used social media. In this study, more than $156(64 \%)$ student showed high interest in receiving COVID-19 related information and support from the university. Students were mainly interested in seeking help with psychosocial support, self-organization and time-management, courses materials, online learning, exam preparations, and COVID-19 proper preventive and protective measures.

\section{Discussion and implications}

In the current study, the mean scores of female students total anxiety, PSS, and IES-R were significantly higher than male students. However, it was reported in another study conducted during the COVID-19 pandemic on university students that there was no gender-related differences between male and female students in terms of stress and negative impact [16]. Our finding is similar to other studies before and after the pandemic. A recent study assessing university students' stress, depression, anxiety, and suicidality in the early stages of the COVID-19 pandemic found that female students scored significantly higher than male students on depression, anxiety, and stress. 
Anxiety and stress levels were higher among women at all study stages which may indicate that these gender differences were present before the pandemic [13]. Another cross-sectional study examined the psychological impact of COVID-19 pandemic on physicians reported that female physicians were more likely to experience fear and worry [21]. In addition, other study reported that work-related stress and anxiety in response to the viral epidemic was related to being female [6]. According to studies conducted even before the pandemic, there is a strong evidence indicates that women, compared with men, report higher levels of anxiety and stress [22, 23] and the pandemic may just confirmed that. We also noticed that junior younger students (18-24 years) scored significantly higher than senior older students ( $\geq 25$ years) on perceived stress $(p<0.02)$ suggesting that younger generations may experience higher levels of stress associated with academic workload and life pressures and are more prone to emotional distress due to lack of knowledge, life experience, and improper coping strategies as reported in other studies [13, 21].

In the current study, having a chronic condition in students was a significant factor that increased anxiety and stress levels of being infected with COVID-19. In another study, chronic condition was associated with elevation in IES-R, depression and anxiety levels [24]. In the current study, living with the family caused a significant decrease in the IES$\mathrm{R}$ total scores in contrast with living with friends or living alone due to family psychological support, bonding, and coherence. However, we found that having family members infected with COVID-19 caused a significant increase in the IES-R scores similar to another study conducted during the same period [16]. We also found that students whose families currently had a lower monthly income than the regular minimum monthly wage prior to the pandemic increased anxiety, perceived stress, and had a negative impact confirming the results of an earlier study in which low family monthly income and financial restrictions during quarantine and COVID-19 pandemic increased sense of danger and anxiety [25].

In this study, a significant number of students reported that they followed COVID-19 updates and relied on valid and reliable resources of information, such as the ministry of health, WHO, and national television. However, almost more than half of the students reported that they used social media as their main source of information similar to other studies findings [26-28]. Furthermore, students with adequate Knowledge about the COVID-19 pandemic who have been utilizing information from valid and reliable official documents and resources reported a significant decrease in anxiety level, perceived stress, and negative impacts of the pandemic (see Table 2) confirming that misconceptions and the spread of misinformation about the COVID-19 pandemic may lead to higher levels of anxiety and stress [29]. In this study, anxiety, PSS and IES-R total scores of junior preclinical students were significantly higher than their senior clinical peers most probably due to the lower knowledge of preclinical students. Clinical students have previous exposure to similar traumatic events, hence were less likely to experience stress and fear. This finding indicates that clinical students have a better awareness of the pandemic's effects on overall health in general and mental health in specific. Thus, university faculty members may offer more support to preclinical students while clinical students can develop support focus groups as suggested by other study [27].

The psychological impact of fear and anxiety induced by the rapid spread of COVID-19 pandemic needs to be clearly recognized as a health priority for regulatory bodies and universities and preventive strategies should be advocated for and adopted to mitigate the impacts of COVID-19 on mental health among public population and university students [30-32]. Implementing psychological interventions and community-based strategies to develop sense of coherence and support resilience and psychologically vulnerable individuals during the COVID-19 crisis are key fundamentals for any community and are playing an important role during COVID-19 pandemic [33-35]. A recent study suggested that resilience and positive coping lead to better psychological and mental health status among students. In contrast, negative coping is a potential risk factor for mental health [15]. The WHO has been working with partners to develop a set of new materials on the mental health and psychosocial support aspects of COVID-19 with emphasis on maintaining a healthy lifestyle and developing support network [10]. Additionally, following specific precautionary measures, such as adhering to environmental health and safety, proper infection control and prevention and social distancing, providing psychologically safe spaces at all community settings, adopting effective self-care strategies and healthy lifestyle behaviors, and encouragement of help-seeking behaviors appear to have a protective effect on the individual's mental health and overall wellbeing [12, 36-38]. Furthermore, 
offering practical support, providing evidence-based scientific information and adequate mental health services, strengthening mental health support systems for medical and rehabilitation professionals and students through blended health education methods, stress management techniques, psychoeducational sessions and online psychological counseling webinars, screening for adverse psychological outcomes, and providing an individualized approach to target the specific fears and detect any warning signs will be crucial to maintain well-being and prevent burnout [39-42]. Such strategies would be helpful, useful, and beneficial in managing symptoms, decreasing negative psychological outcomes, adapting and coping with COVID-19 related stress, and maintaining a mentally healthier world [4, 43-46]. Highlighting the positive aspects of the current situation, such as building family and friends support systems, strengthening family members bonding, utilizing technological tools to implement effective human communication and interpersonal relationships, enhancing role of empathy and telehealth services, and adopting healthy alternative habits and routines are key and crucial [47]. Maintaining a positive state of mind and positive way of thinking helps increase sense of hope and optimism to those who are fearful [48, 49]. Universities and families need to enhance students' knowledge and awareness and closely monitor and promote healthy behaviors through continuous education and support [50].

\subsection{Implications}

This is the first national study examining the mental wellbeing and support needs following the COVID19 pandemic for medical and rehabilitation sciences students in Saudi Arabia. The results of this study may have regional, national, and international implications and could be used to assist medical and rehabilitation sciences schools and clinical training settings in decision and policy making. We hope that this study will help facilitate the developments of tailored prevention and intervention programs to mitigate the impact of the pandemic on mental health and wellbeing and will have a positive significant impact on students overall health and academic performance.

\subsection{Limitations}

Although this study is not free from limitations, this was the first national study examining the mental wellbeing and support needs following the
COVID-19 pandemic for medical and rehabilitation sciences students in Saudi Arabia. In addition, our findings provide insights into stress and mental health among university students during the COVID-19 pandemic. We hope that this study will help understand the stress experienced by medical and rehabilitation sciences students and findings can be used for a more effective identification of students who might be at risk and may struggle during next stages of the pandemic and future crises. The current study was conducted in only one medical school. Therefore, it may not represent all medical and rehabilitation sciences schools. Furthermore, participation in the research was voluntary, all students were not available and we had limited access to them due to other curricular demands and university workloads. For these reasons, some students did not participate in the study. For future studies, a larger sample through the inclusion of more universities on a national scale and students from other medical and rehabilitation programs is crucial and recommended.

\section{Conclusions}

This study investigated the psychological effects of the COVID-19 pandemic on medical and rehabilitation sciences university students and found that they were highly anxious about being infected with COVID-19 especially among female students. The first hypothesis that medical and rehabilitation sciences students are highly anxious about being infected with COVID-19, therefore, they are at increased risk of developing psychological and mental health issues during the COVID-19 crisis was totally confirmed especially among female students. The second hypothesis that preclinical students have less knowledge than clinical students, hence will have a significant higher levels of anxiety and stress was also totally confirmed. The current study shed light on important factors that play a major role in anxiety, perceived stress and impact of COVID-19 pandemic among medical and health sciences university students, such as gender differences, presence of chronic condition, knowledge base, family monthly income and financial status, and students educational level and stage (preclinical and clinical).

In order to support medical and rehabilitation sciences university students appropriately both now and, in the future, it is imperative that we understand the impact it has had upon them. Medical and rehabilitation sciences students are at increased risk of 
developing psychological and mental health issues during the COVID-19 crisis. Special supportive measures should be implemented during and after the pandemic is over. Further research on the risk and preventive factors should be taken into account in future. Developing proper mental health management guidelines and providing psychological support, psychosocial crisis prevention and counseling services at both the university and community levels are highly recommended and should be considered.

\section{Conflict of interest}

The author declared no potential conflicts of interests with respect to the research, authorship, and/or publication of this article.

\section{References}

[1] Brooke J, Jackson D. Older people and COVID-19: Isolation, Risk and Ageism. 2020

[2] Dubey S, Biswas P, Ghosh R, Chatterjee S, Dubey MJ, Chatterjee S, Lahiri D, Lavie CJ. Psychosocial impact of COVID-19. Diabetes \& Metabolic Syndrome: Clinical Research \& Reviews. 2020;14(5):779-88.

[3] Jimeno JP. Psychological effects of COVID-19. Archivos De La Sociedad Espan ola De Oftalmologi' a. 2020 Sep 1.

[4] Ng KY, Zhou S, Tan SH, Ishak ND, Goh ZZ, Chua ZY, Chia JM, Chew EL, Shwe T, Mok JK, Leong SS. Understanding the Psychological Impact of COVID-19 Pandemic on Patients With Cancer, Their Caregivers, and Health Care Workers in Singapore. JCO Global Oncology. 2020;6: 1494-509.

[5] Lai J, Ma S, Wang Y, Cai Z, Hu J, Wei N, Wu J, Du H, Chen T, Li R, Tan H. Factors associated with mental health outcomes among health care workers exposed to coronavirus disease 2019. JAMA Network Open. 2020;3(3):e203976.

[6] Lee JK, Lee HJ, Hong Y, Shin YW, Chung S, Park J. The Hazardous Workplace, Work-related Stress, and Unhealthy Behaviors among Healthcare Workers: The Relationships with Depressive and Insomnia symptoms during COVID-19.

[7] Wong LP, Hung CC, Alias H, Lee TS. Anxiety symptoms and preventive measures during the COVID-19 outbreak in Taiwan. BMC Psychiatry. 2020;20(1):1-9.

[8] Zaki NF, Sidiq M, Qasim M, Aranas B, Hakamy A, Ruwais NA, Alanezi H, Al Saudi DA, Alshahrani RB, Al-Thomali AB, Manzar MD. Stress and psychological consequences of COVID-19 on health-care workers. Journal of Nature and Science of Medicine. 2020;3(4):299.

[9] Ismael F, Bizario JC, Battagin T, Zaramella B, Leal FE, Torales J, Ventriglio A, Marziali ME, Martins SS, Castaldelli-Maia JM. Post-infection depression, anxiety and PTSD: a retrospective cohort study with mild COVID-19 patients. medRxiv. 2020 Jan 1.
[10] Kaur H, Mahajan P. Library and Information Services of AC Joshi Library during COVID-19 pandemic: a study of faculty satisfaction. Library Philosophy and Practice. 2020:1-20.

[11] Ma ZF, Zhang Y, Luo X, Li X, Li Y, Liu S, Zhang Y. Increased stressful impact among general population in mainland China amid the COVID-19 pandemic: a nationwide cross-sectional study conducted after Wuhan city's travel ban was lifted. International Journal of Social Psychiatry. 2020;66(8):770-9.

[12] Alkhamees AA, Alrashed SA, Alzunaydi AA, Almohimeed AS, Aljohani MS. The psychological impact of COVID19 pandemic on the general population of Saudi Arabia. Comprehensive Psychiatry. 2020;102:152192.

[13] Debowska A, Horeczy B, Boduszek D, Dolinski D. A repeated cross-sectional survey assessing university students' stress, depression, anxiety, and suicidality in the early stages of the COVID-19 pandemic in Poland. Psychological Medicine. 2020:1-4.

[14] Akat M, Karataş K. Psychological Effects of COVID-19 Pandemic on Society and Its Reflections on Education. Electronic Turkish Studies. 2020;15(4).

[15] Zhang C, Ye M, Fu Y, Yang M, Luo F, Yuan J, Tao Q. The psychological impact of the COVID-19 pandemic on teenagers in China. Journal of Adolescent Health. 2020; 67(6):747-55

[16] Cao W, Fang Z, Hou G, Han M, Xu X, Dong J, Zheng J. The psychological impact of the COVID-19 epidemic on college students in China. Psychiatry Research. 2020;287:112934.

[17] Kim JS, Choi JS. Middle East respiratory syndrome-related knowledge, preventive behaviours and risk perception among nursing students during outbreak. Journal of Clinical Nursing. 2016;25(17-18):2542-9.

[18] Cohen S, Kamarck T, Mermelstein R. A global measure of perceived stress. Journal of Health and Social Behavior. 1983:385-96.

[19] Lee EH. Review of the psychometric evidence of the perceived stress scale. Asian Nursing Research. 2012; 6(4):121-7.

[20] Christianson S, Marren J. The impact of event scale-revised (IES-R). Medsurg Nursing. 2012;21(5):321-3.

[21] Al Sulais E, Mosli M, AlAmeel T. The psychological impact of COVID-19 pandemic on physicians in Saudi Arabia: A cross-sectional study. Saudi Journal of Gastroenterology: Official Journal of the Saudi Gastroenterology Association. 2020;26(5):249.

[22] Gentry LA, Chung JJ, Aung N, Keller S, Heinrich KM, Maddock JE. Gender differences in stress and coping among adults living in Hawaii. Californian Journal of Health Promotion. 2007;5(2):89-102.

[23] McLean CP, Anderson ER. Brave men and timid women? A review of the gender differences in fear and anxiety. Clinical Psychology Review. 2009;29(6):496-505.

[24] Wang C, Pan R, Wan X, Tan Y, Xu L, Ho CS, Ho RC. Immediate psychological responses and associated factors during the initial stage of the 2019 coronavirus disease (COVID-19) epidemic among the general population in China. International Journal of Environmental Research and Public Health. 2020;17(5):1729.

[25] Sallam M, Dababseh D, Yaseen A, Al-Haidar A, Ababneh NA, Bakri FG, Mahafzah A. Conspiracy beliefs are associated with lower knowledge and higher anxiety levels regarding COVID-19 among students at the University of Jordan. International Journal of Environmental Research and Public Health. 2020;17(14):4915. 
[26] Ikhlaq A, Hunniya BE, Riaz IB, Ijaz F. Awareness and attitude of undergraduate medical students towards 2019novel corona virus. Pakistan Journal of Medical Sciences. 2020;36(COVID19-S4):S32.

[27] Taghrir MH, Borazjani R, Shiraly R. COVID-19 and Iranian medical students; a survey on their related-knowledge, preventive behaviors and risk perception. Archives of Iranian Medicine. 2020;23(4):249-54.

[28] Sahin SK, Arslan E, Atalay ÜM, Demir B, Elboga G, Altındağ A. Psychological impact of COVID-19 outbreak on health workers in a university hospital in Turkey. Psychology, Health \& Medicine. 2021:1-0.

[29] Mian A, Khan S. Coronavirus: the spread of misinformation. BMC Medicine. 2020;18(1):1-2.

[30] Aylie NS, Mekonen MA, Mekuria RM. The psychological impacts of COVID-19 pandemic among university students in Bench-Sheko Zone, South-west Ethiopia: a communitybased cross-sectional study. Psychology Research and Behavior Management. 2020;13:813.

[31] Delmastro M, Zamariola G. The Psychological Effect of COVID-19 and Lockdown on the Population: Evidence from Italy.

[32] Banerjee D, Vaishnav M, Rao TS, Raju MS, Dalal PK, Javed A, Saha G, Mishra KK, Kumar V, Jagiwala MP. Impact of the COVID-19 pandemic on psychosocial health and wellbeing in South-Asian (World Psychiatric Association zone 16) countries: A systematic and advocacy review from the Indian Psychiatric Society. Indian Journal of Psychiatry. 2020;62(Suppl 3):S343.

[33] Renjun G, Ziyun L, Xiwu Y, Wei W, Yihuang G, Chunbing Z, Zhiguang S. Psychological intervention on COVID-19: a protocol for systematic review and meta-analysis. Medicine. 2020;99(21).

[34] Schäfer SK, Sopp MR, Schanz CG, Staginnus M, Göritz AS, Michael T. Impact of COVID-19 on public mental health and the buffering effect of a sense of coherence. Psychotherapy and psychosomatics. 2020;89(6):386-92.

[35] Serafini G, Parmigiani B, Amerio A, Aguglia A, Sher L, Amore M. The psychological impact of COVID-19 on the mental health in the general population. QJM: An International Journal of Medicine. 2020;113(8):531-7.

[36] AlHumaid J, Ali S, Farooq I. The psychological effects of the COVID-19 pandemic and coping with them in Saudi Arabia. Psychological Trauma: Theory, Research, Practice, and Policy. 2020;12(5):505.

[37] Blake H, Bermingham F, Johnson G, Tabner A. Mitigating the psychological impact of COVID-19 on healthcare workers: a digital learning package. International journal of environmental research and public health. 2020;17(9):2997.

[38] Paraskevopoulos E, Papandreou M. Systematic infection control in Greek Physiotherapy Practices during the COVID-19 pandemic. Work (Reading, Mass.). 2020;66(2): $367-70$.
[39] Ahn MH, Shin YW, Suh S, Kim JH, Kim HJ, Lee KU, Chung S. High work-related stress and anxiety response to COVID-19 among healthcare workers in South Korea: save study.

[40] Balhareth A, AlDuhileb MA, Aldulaijan FA, Aldossary MY. Impact of COVID-19 pandemic on residency and fellowship training programs in Saudi Arabia: A nationwide cross-sectional study. Annals of Medicine and Surgery. 2020;57:127-32.

[41] Sritharan J, Jegathesan T, Vimaleswaran D, Sritharan A Mental Health Concerns of Frontline Workers During the COVID-19 Pandemic: A Scoping Review. Global Journal of Health Science. 2020;12(11):89-105.

[42] Abbasi MS, Ahmed N, Sajjad B, Alshahrani A, Saeed S, Sarfaraz S, Alhamdan RS, Vohra F, Abduljabbar T. E-Learning perception and satisfaction among health sciences students amid the COVID-19 pandemic. Work. 2020;67(3):549-56.

[43] Cunill Olivas M, Aymerich Andreu M, Serdà Ferrer BC, Patiño Masó J. The Impact of COVID-19 on Spanish Health Professionals: A Description of Physical and Psychological Effects. International Journal of Mental Health Promotion, 2020, vol. 22, núm. 3, p. 185-198. 2020 Aug 14.

[44] Marshall JM, Dunstan DA, Bartik W. The role of digital mental health resources to treat trauma symptoms in Australia during COVID-19. Psychological Trauma: Theory, Research, Practice, and Policy. 2020 Jun 4.

[45] Si MY, Su XY, Jiang Y, Wang WJ, Gu XF, Ma L, Li J, Zhang SK, Ren ZF, Ren R, Liu YL. Psychological impact of COVID-19 on medical care workers in China. Infectious diseases of poverty. 2020;9(1):1-3.

[46] Sugaya N, Yamamoto T, Suzuki N, Uchiumi C. A realtime survey on the psychological impact of mild lockdown for COVID-19 in the Japanese population. Scientific Data. 2020;7(1):1-6.

[47] Bandyopadhyay S, Georgiou I, Baykeens B, Gillespie CS, de Andres Crespo M, Bashir MT, Handa A, Saunders KE. Medical students' mood adversely affected by COVID-19 pandemic: An interim analysis from the SPICE-19 prospective cohort study of 2075 medical students and interim foundation doctors.

[48] Chawla N, Sharma P, Sagar R. Psychological Impact of COVID-19 on Children and Adolescents: Is There a Silver Lining?. The Indian Journal of Pediatrics. 2021 Jan;88(1):91-.

[49] Saladino V, Algeri D, Auriemma V. The psychological and social impact of Covid-19: new perspectives of well-being. Frontiers in psychology. 2020;11:2550.

[50] Zandian H, Sarailoo M, Dargahi S, Gholizadeh H, Vosoughi M, Dargahi A. Evaluation of knowledge and health behavior of university of medical sciences students about the prevention of COVID-19. Work. 2021;68(3):543-49. 